\title{
Of talmomiíase interna posterior: relato de dois casos de larva viva no espaço sub-retiniano
}

\author{
Internal posterior ophthalmomyiasis: report of two cases oflive subretinal larva
}

\author{
Rogiil José de Almeida Torres ${ }^{1}$ \\ Naoye Shiokawa ${ }^{2}$ \\ Rog'ério João de Almeida Torres ${ }^{3}$
}

Trabalho realizado no Centro Oftalmológico de Curitiba. ${ }^{1}$ Médico oftalmologista. Ex-fellow de retina e vítreo do Instituto Penido Burnier. Retinólogo do Centro Oftalmológico de Curitiba.

${ }^{2}$ Professor Adjunto da Universidade Federal do Paraná. Chefe do departamento de retina e vítreo da Oftalmoclínica Curitiba.

${ }^{3}$ Médico oftalmologista. Ex-residente do Instituto Penido Burnier.

Endereço para correspondência: Praça Rui Barbosa, 827 - Conj. 305 - Curitiba (PR) CEP 80010-030.

\begin{tabular}{|l|}
\hline \multicolumn{1}{|c|}{ RESUMO } \\
\hline $\begin{array}{l}\text { Descrevemos dois casos de oftalmomiíase interna posterior, com a } \\
\text { presença de larva viva no espaço subretiniano. Fazemos uma revisão } \\
\text { bibliográfica, dando ênfase ao diagnóstico e tratamento desta rara } \\
\text { afecção ocular infecciosa. }\end{array}$
\end{tabular}

Descritores: Miíase/diagnóstico; Fotocoagulação; Infecções oculares parasitárias; Muscidae/parasitologia; Relato de caso

\section{INTRODUÇÃ̃O}

O termo oftalmomiíase ou miíase ocular significa infestação do olho pelo estágio larval de certas moscas da ordem díptera. Esta afecção é considerada rara e ocorre em menos de $5 \%$ dos casos de miíase humana ${ }^{(1)}$. Classificamos esta doença, de acordo com a localização da larva, nas formas orbitária, interna e externa ${ }^{(1)}$. A presença, no interior do bulbo ocular, caracteriza a oftalmomiíase interna. Os ovos ou larvas são transportados para a superfície ocular, córnea ou conjuntiva, pela mosca adulta, por um vetor secundário ou pelas mãos do paciente. Perfuram estas camadas oculares e ganham o espaço intra-ocular, podendo se localizar na câmara anterior, câmara posterior, vítreo e espaço sub-retiniano ${ }^{(2-3)}$. As larvas responsáveis pela afecção do bulbo ocular normalmente são provenientes de moscas de gado (gênero hipoderma), porém já foram relatados casos de larvas de moscas de cavalo (gênero Oedemagena tarandi e Cuterebes) ${ }^{(3-4)}$. São parasitas obrigatórios, necessitando de tecido de hospedeiro vivo para completar seu estágio larval $^{(1,5)}$. O paciente pode ser assintomático mas também pode exibir queda da acuidade visual por uveíte, vitreíte ou outras complicações coriorretinianas. O diagnóstico pode ser feito pela observação do traçado no espaço sub-retiniano ou pela identificação direta da larva, que é semi-translúcida, segmentada e afilada em suas extremidades. O objetivo deste trabalho é apresentar dois casos raros de oftalmomiíase interna posterior em que as larvas encontravam-se vivas, no espaço sub-retiniano.

\section{APRESENTAÇÃO DE CASOS}

Caso 1: C.L.F.P., sexo masculino, 28 anos de idade, servente, natural e procedente de Campo Largo (PR). Veio a primeira consulta em 19/04/93, relatando diminuição abrupta da acuidade visual de olho esquerdo (O.E.) há 3 meses. Ao exame oftalmológico constatou-se: acuidade visual com correção (A.V. c/c) de O.D.=1,0 (20/20) e de O.E.= conta dedos a 4 metros. A biomicroscopia de ambos os olhos (A. O.) mostrou-se sem alterações. A 
oftalmoscopia direta de O.E. demonstrou presença de linha branco-amarelada acima do disco óptico, estendendo-se ao feixe papilo-macular, atravessando a região foveolar e interrompendo-se temporalmente à fóvea. Nesta extremidade, havia discreto aumento do diâmetro do traçado. A oftalmoscopia binocular indireta revelou que este traçado alcançava a região próxima a ora serrata, às 12 horas, possuindo trajetória curvilínea. Na região mais periférica predominava pigmentação, tornando-se despigmentada no polo posterior (Figura 1). À retinografia fluorescente, o setor mais periférico do traçado mostrou-se hipofluorescente, devido ao bloqueio da fluorescência de fundo ocular, ocasionado pelo acúmulo de pigmentos. Na região próxima ao polo posterior predominou a fluorescência, devido à atrofia do EPR. Foi detectado também, à angiografia fluoresceínica, membrana neovascular sub-retiniana em região foveolar. Solicitamos hemograma e ELISA para toxocaríase que resultaram respectivamente: normal e não reagente. Outra retinografia foi realizada, a qual demonstrou avanço da lesão (Figura 2). Foi indicada e realizada fotocoagulação, com laser de argônio, usando mira de aproximadamente 400 micra, com duração de 0,3 segundo e intensidade que variou de 400 a $600 \mathrm{~mW}$, objetivando matar a larva. Dois anos após, observamos pigmentação de todo o traçado sub-retiniano e aumento da membrana neovascular. Não houve extensão do traçado.

Caso 2: Z. M. R., 42 anos, sexo feminino, natural e procedente de São Joaquim (SC). Veio à primeira consulta no dia 24/ 7/86, queixando-se de metamorfopsia em O.E. Ao exame oftalmológico constatou-se: A.V. c/c de A.O.=1,0 (20/20). Biomicroscopia de A. O. sem alterações nas câmaras anteriores e vítrea (ausência de sinais inflamatórios). A oftamoscopia e a retinografia aneritra demonstraram presença de larva viva e móvel no espaço sub-retiniano em região macular (Figuras $3 \mathrm{e}$ 4). Foi indicada a fotocoagulação, com laser de argônio, da larva com o intuito de matá-la, porém tal procedimento não foi

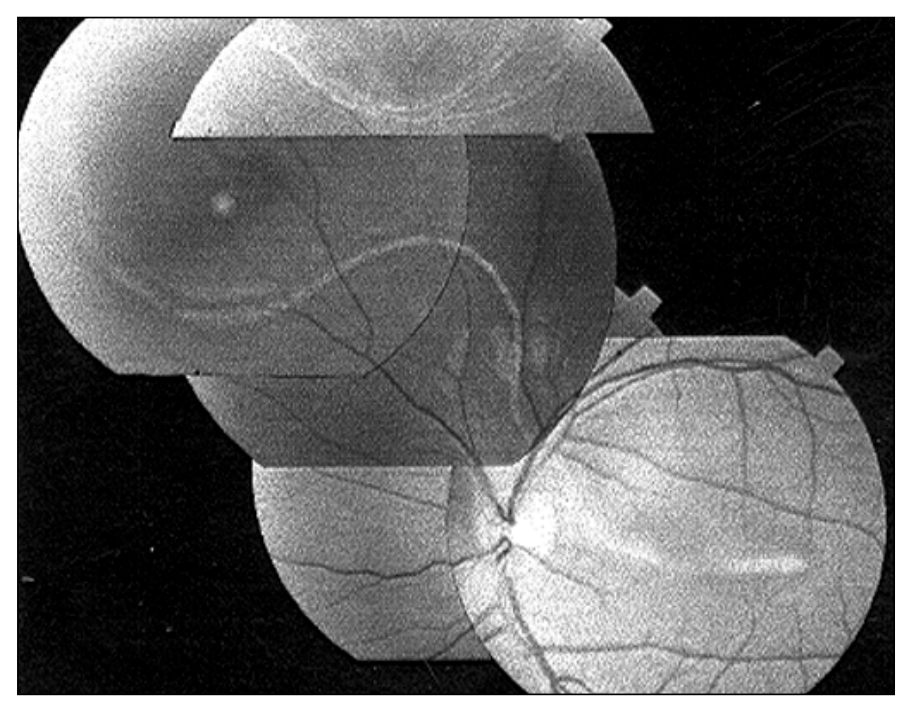

Figura 1 - Caso 1: Montagem fotográfica do fundo ocular demonstrando a trilha deixada pela larva. Este traçado é considerado patognomônico de miíase sub-retiniana aceito. A paciente retornou no dia $01 / 06 / 89$, queixando-se de diminuição da acuidade visual de O.E. há 2 meses. O exame de fundo de olho demonstrou perda do reflexo foveolar e focos hemorrágicos sub-retinianos na região macular. $\mathrm{O}$ exame de biomicroscopia posterior de O.E. revelou elevação da retina sensorial em região macular. A angiografia fluoresceínica confirmou a presença de membrana neovascular sub-retiniana.

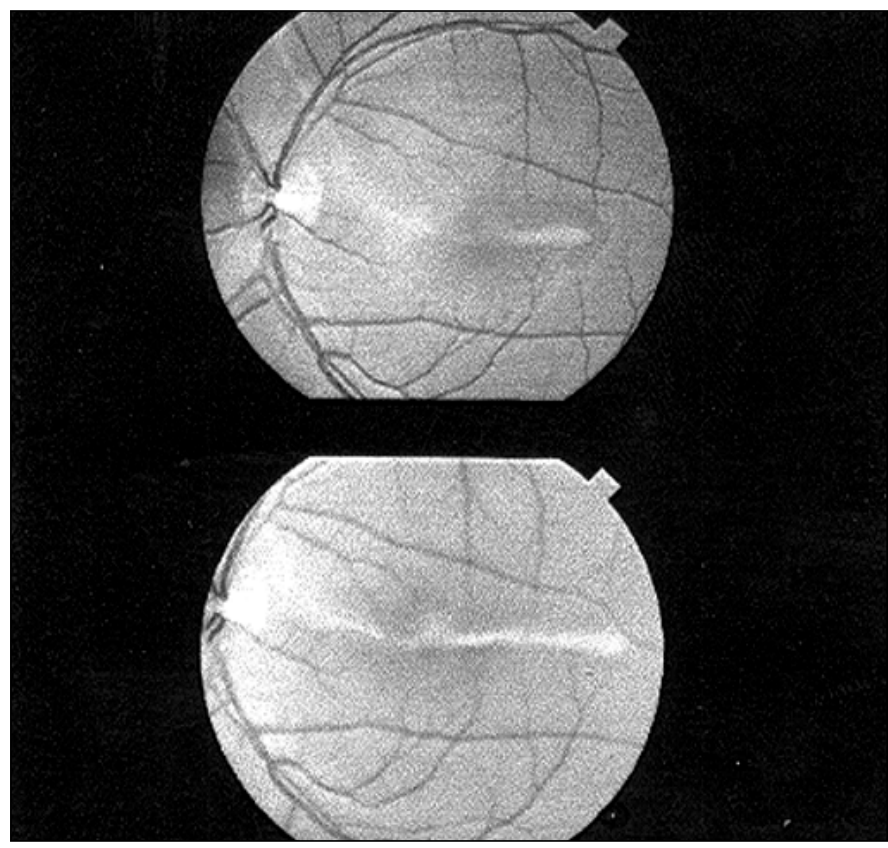

Figura 2 - Caso 1: Retinografia superior realizada na primeira consulta. Retinografia inferior realizada após 1 mês. Comparando as duas fotografias observamos o avanço da lesão

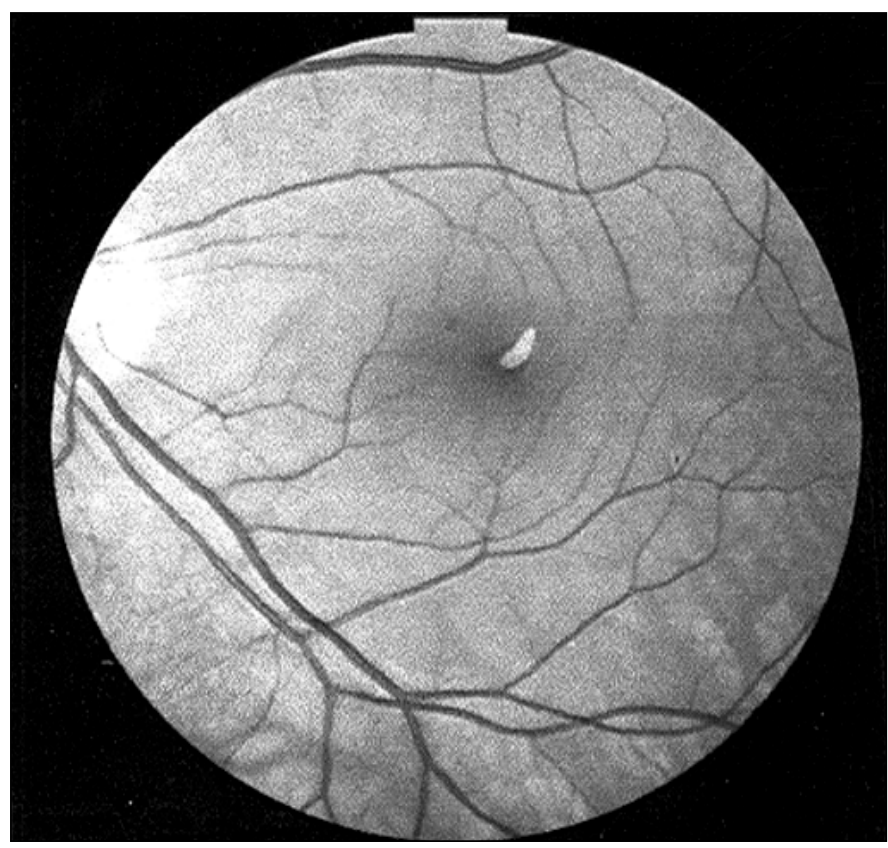

Figura 3 - Caso 2: Retinografia demonstrando a presença de larva subretiniana semi-translúcida, segmentada e afilada em suas extremidades 


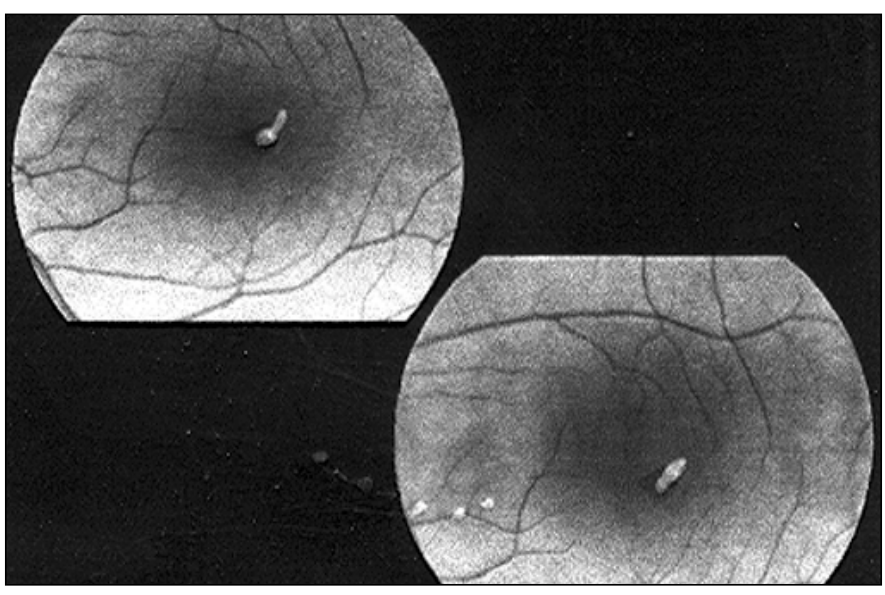

Figura 4 - Caso 2: Retinografias aneritras demonstrando alterações morfológicas da larva indicando sua mobilidade no espaço subretiniano

\section{DISCUSSÃO}

No primeiro caso, os achados clínicos, fundoscópicos e angiográficos, sugeriram que havia algum organismo móvel entre a retina e o EPR, deixando uma trilha. Este traçado corresponde ao modelo, cor e tamanho observado por Dixon ${ }^{(3)}$, Hunt $^{(7)}$, Fitzgeral e Rubin ${ }^{(6)}$, sendo considerado patognomônico de miíase sub-retiniana. Estas alterações do EPR podem ser causadas pela fricção do movimento da larva, pela alimentação, pelos produtos tóxicos ou pela combinação destes fatores. No segundo caso, não havia este traçado. A observação fundoscópica e angiográfica da larva, viva e móvel, no espaço sub-retiniano, possibilitou o diagnóstico. Sabe-se que a larva, eventualmente, pode migrar pela coróide, sem deixar sinais ${ }^{(8)}$. Em ambos os casos a condição foi unilateral. A ausência de eosinofilia e o teste de ELISA não reagente para toxocaríase, contribuiram para afastar outras possíveis etiologias. Os pacientes eram assintomáticos e queixaram-se somente quando a região macular foi afetada, exibindo baixa considerável da visão no primeiro caso e metamorfopsia no segundo caso. Não foi possível identificar o local de entrada da larva. Segundo Dixon e Hunt ${ }^{(3,7)}$, nesta área ocorreria hiperplasia pigmentária reativa, devido à formação de pequenas hemorragias, marcando o local de penetração da larva através da coróide. No primeiro caso o traçado estendeu-se até a região próxima à ora serrata, às 12 horas, porém sem acúmulo excessivo de pigmentos mas com regularidade de pigmentação em relação aos demais pontos do trajeto. No segundo caso, as alterações ocasionadas pela larva estavam restritas ao polo posterior, não havendo alterações pigmentárias em outras partes da retina. Nos dois casos, as larvas migraram para a mácula, fator indicativo de que esta seja a região mais apropriada, dentro do olho, para a larva se localizar. Neste setor, a taxa de oxigenação sanguínea é maior que em outras partes da retina. A formação de membrana neovascular sub-retiniana, em região macular, foi a complicação comum observada em ambos pacientes. Provavelmente ocorreu rompimento da membrana de Brüch, possibilitando a proliferação neovascular, proveniente da coróide, para o espaço sub-retiniano. Até 1982, dos 31 casos relatados, somente em 6 olhos a larva encontrava-se viva. Nos dois casos que apresentamos, as larvas encontravam-se vivas no espaço sub-retiniano. No primeiro, as angiografias realizadas em períodos diferentes, demonstraram a evolução do traçado sub-retiniano. No segundo, a larva apresentava movimentos contínuos no espaço sub-retiniano que eram facilmente notados à fundoscopia.

$\mathrm{Na}$ literatura nacional Esteves e Maestri publicaram um caso de oftalmomíase interna posterior presumida ${ }^{(9)}$.

Quanto ao tratamento propõem-se que: $1^{\circ}$ ) Se a larva estiver morta e não houver reação inflamatória, convém adotar conduta expectante $\left.{ }^{(5,10)} \cdot 2^{\circ}\right)$ Se a larva estiver viva no vítreo há autores que defendem a vitrectomia posterior seguida de sua remoção ${ }^{(5)}$, ou observação, caso não haja reação inflamatória ${ }^{(4-5)} \cdot 3^{\circ}$ ) Se a larva estiver viva no espaço sub-retiniano devemos destruí-la pela fotocoagulação ${ }^{(5,7,10)}$. No primeiro caso, foi possível eliminar a larva pela fotocoagulação. No segundo caso, a paciente recusou o tratamento a laser, provavelmente porque sua visão central era relativamente boa e a metamorfopsia não a incomodava suficientemente, para aceitar tal tratamento.

\section{ABSTRACT}

We describe two cases of internal posterior ophthalmomyiasis with the presence of live subretinal larva. We present a review of previously reported cases, emphasizing the diagnosis and treatment of this rare ocular infection.

Keywords: Myasis/diagnosis; Light coagulation; Parasitic eye infections; Muscidae/parasitology; Case report

\section{REFERÊNCIAS}

1. Kean BH, Sun T, Ellsworth RM. Text of ophthalmic parasitology. New York: Hardcover; 1991. p. 222-8.

2. Duke-Elder WS. Endovascular myasis. In: Duke-Elder WS. System of ophthalmology. St. Louis: Mosby Year Book; 1996. p. 490-4.

3. Dixon JM, Winkler CH, Nelson JH. Ophthalmomyiasis interna caused by Cuterebra larva. Am J Ophthalmol 1971; 1(1 Part 2):415-6.

4. Syrdalen P, Nitter T, Mehl R. Ophthalmomyiasis interna posterior: report of case caused by the reindeer warble fly larva and review of previous reported cases. Br J Ophthalmol 1982;66:589-93.

5. Gass JD, Lewis RA. Subretinal tracks in ophthalmomyiasis. Arch Ophthalmol 1976;94:1500-5.

6. Fitzgerald CR, Rubin ML. Intraocular parasite destroyed by photocoagulation. Arch Ophthalmol 1974;91:162-4.

7. Hunt Jr. EW. Unusual case of ophthalmomyiasis interna posterior. Am J Ophthalmol 1970;70:978-80.

8. Mason GI. Bilateral ophthalmomyiasis interna. Am J Ophthalmol 1981;91:65-70.

9. Ziemianski MC, Lee Ky, Sabates FN. Ophthalmomyiasis interna. Arch Ophthalmol 1980;98:1588-9.

10. Esteves JF, Maestri MK. Oftalmomiíase sub-retiniana presumida. Arq Bras Oftalmol 1993;56:62-4. 